\title{
Identification of ankylosing spondylitis-associated genes by expression profiling
}

\author{
HUI MA ${ }^{*}$, DABO XU* and QIANG FU \\ Department of Orthopaedics, Changhai Hospital, Second Military Medical University, Shanghai, P.R. China
}

Received March 2, 2012; Accepted May 11, 2012

DOI: $10.3892 / \mathrm{ijmm} .2012 .1047$

\begin{abstract}
Ankylosing spondylitis (AS) is a chronic inflammation attacking the sacroiliac joints and the spine. Certain genes have been associated with the occurrence of AS Gene chip data were utilized to recognize genes associated with AS for the association of the clinical diagnosis and the biomedical study. Microarray expression data of AS were acquired from the public microarray database GEO (gene expression omnibus), and AS-related genes were obtained by differential gene expression profiling. The transcriptional and translational levels of these genes were further examined. The transcriptional and translational levels of three genes were shown to be upregulated in a mouse model of AS by real-time PCR and ELISA assay, respectively. Differential expression of AS-related genes was identified by analysis of gene chip data, contributing to the advancement of the understanding of the pathogenesis of AS.
\end{abstract}

\section{Introduction}

Ankylosing spondylitis (AS), a chronic inflammation attacking sacroiliac joints and the spine, is a complex genetic disease (1) with $3 \%$ incidence rate in yellow race $(2,3)$. Although the exact cause of AS is still unclear, there is a close relationship between the genetic information and the occurrence of AS. Studies show that the major histocompatibility complex region (MHC) is closely related to AS, and about 95\% AS patients are born with a gene named HLA-B27. However, not only the HLA-B27 gene, but also other important genes are believed to participate in the process of AS (4). In addition, numerous AS-related genes remain to be identified.

Gene expression profiling analysis is a bioinformatics approaches whose application is rising in recent years. This

Correspondence to: Dr Qiang Fu, Department of Orthopaedics, Changhai Hospital, Second Military Medical University, Changhai Road 168, Shanghai 200433, P.R. China

E-mail: qingfuqf@hotmail.com

*Co-first authors

Key words: gene expression profiling, ankylosing spondylitis, RT-PCR, ELISA approach can perform high throughput analysis of thousands of genes at one time. The combination of gene expression profiling and disease genomics results in deep understanding of the relationship of hereditary disease and genes, which has become an important research direction.

There are many ethical issues regarding the performance of experimental assays in humans. Our study utilized the AS mouse model for the research of the disease (5). First, the gene expression data were integrated into the public Gene Expression Omnibus (GEO), and bioinformatics analyses were performed to screen the potential disease-associated genes in a large scale. Blood samples of normal and AS model mice before and after treatment of TNF- $\alpha$ were gathered, and RT-PCR and ELISA assays, were carried out to examine tehe mRNA and protein levels of the genes, respectively. The association of the potential gene with the disease was assesssed by examining the transcriptional and translational levels of the genes, and by functional analysis of one gene. This study provides novel insights into the diagnosis, prevention and treatment of the disease.

\section{Materials and methods}

Screening of differentially expressed AS-related genes. Gene chip data GSE25101 were downloaded from the United States National Center for Biotechnology Information Sharing database GEO, and analyzed by the Vizard 1.2 software. After signal extraction, normalization and chip comparison analysis, the AS-related genes were screened according to the ratio values. Through gene ontology (GO) analysis performed on the http://www.gosurfer.org website, the molecular function, biological pathways and cellular components of differentially expressed genes were obtained. The target genes associated with AS were screened by combination of the pathophysiology process of disease and the related study.

Establishment of the AS mouse model. The AS mouse model was established with a transgenic method. The plasmid was constructed by our laboratory, and the Kunming mice were purchased from the Institute of Experimental Animals, Chinese Academy of Medical Sciences. Development of the mouse model has been previously described. The animals that were successfully converted into AS model mice were administered the TNF inhibitor etanercept (Shanghai CITIC Health Pharmaceutical Co., Ltd) for over 6 months (1). 
Genomic DNA extraction. Venous blood (1 ml) was taken from normal and AS model mice, and $0.4 \mathrm{ml}$ anticoagulants was added to extract genomic DNA with the high salt precipitation method (blood was taken from model mice before and after treatment). The DNA concentration was obtained with the NanoDrop 2000 spectrophotometer (Thermo Scientific). The DNA samples were conserved at $-80^{\circ} \mathrm{C}$.

Detection of gene transcription levels. According to the screened genes in step 1, real-time PCR primers were designed using the online website http://www.idtdna.com. The relative expression quantity of the amplified target gene and 18sRNA gene fragments were detected with SYBR-Green I dye method. The PCR conditions were $95^{\circ} \mathrm{C}$ for $3 \mathrm{sec}, 60^{\circ} \mathrm{C}$ for $5 \mathrm{sec}, 72^{\circ} \mathrm{C}$ for $30 \mathrm{sec}$, for 40 cycles. Real-time detection of fluorescence intensity in each cycle during the PCR reaction process was obtained to achieve equivalent detection. The standard curve of the respective standard samples were established, and the relative expression levels of the target genes in the control group and the AS model group after treatment were analyzed based on the control gene of 18sRNA. All experimental date were statistically analyzed by SPSS 15.0 software, and data were obtained from the software in the CFX-96 real-time quantitative PCR instrument (Bio-Rad) with One-way ANOVA variance analysis. $\mathrm{P}<0.05$ represents a significant difference, and $\mathrm{P}<0.01$ represents a very significant difference.

Examination of protein expression levels. The protein expression levels of the screened genes were examined with ELISA kits (Biosource) in the serum according to the manufacturer's instructions.

Functional analysis of the target gene cytotoxic T lymphocyte-associated antigen 4 (CTLA4). T lymphocytes were isolated from peripheral blood with immunomagnetic beads, and then cultured in RPMI-1640 medium supplemented with $10 \%$ fetal bovine serum in 24 -well plates with $5 \times 10^{5}$ cells in each well. The cells were divided into 5 groups. Each groups was treated with $30,60,90$ or $120 \mathrm{ng} / \mathrm{ml} \mathrm{CTLA} 4$ and the saline group was used as the control. After $48 \mathrm{~h}$, each group of cells was harvested, and the levels of interleukin 5 (IL-5) in the supernatant were detected with the ELISA kit (Biosource).

\section{Results}

Screening of differentially expressed genes. Through screening differentially expressed genes, a total of 83 AS-associated genes were obtained, of which 74 genes were upregulated and 9 genes were downregulated. Differential genes related to AS are involved in immunity, cell cycle, apoptosis, differentiation, signal transduction, cell activity, cell structure, development, stress response, transcription regulation, metabolism and other processes. The results of the present study showed that the molecular function and biological pathway of 13 genes participated in the inflammatory and immune responses, accounting for $15.7 \%$ (Table I).

Based on the microarray results, disease pathophysiology and former research, we further embarked on a clinical study, and screened target genes associated with AS. A total of 3
Table I. Differentially expressed genes associated with AS.

\begin{tabular}{lc}
\hline Gene & Fold-difference \\
\hline ITGA6 & 1.5 \\
PSMB9 & 0.8 \\
TRAT1 & 1.6 \\
PHACTR1 & 1.6 \\
CTLA4 & 1.5 \\
BIRC3 & 1.3 \\
THBD & 1.4 \\
IL-15 & 1.8 \\
BCL11A & 0.9 \\
NKG7 & 1.5 \\
VNN1 & 1.2 \\
MRPL43 & 1.4 \\
PGGT1B & 1.4 \\
\hline
\end{tabular}

target genes were identified: cell receptor related to membrane ligands (TRAT1), CTLA4, IL-15.

Establishment of the AS mouse model. Three hundred Kunming mice were injected with hormones for superovulation, and fertilized eggs were acquired. Genomic DNA fragments containing the HLA-B2704 gene were microinjected into the zygote pronuclear, and two-cell stage fertilized eggs that survived were further transferred to the fallopian tube in pseudopregnant animals to produce the offspring. The expression level of the HLA-B2704 gene in the blood was detected by reverse transcription PCR (Fig. 1). Ten positive mice were successfully obtained, and the AS mouse model was established.

Transcription level of differentially expressed gene. An online primer design website were used to design primers of the 3 screened genes, namely TRAT1, CTLA4 and IL-15, with the housekeeping gene $18 \mathrm{~S}$ as a reference. The primer sequences are shown in Table II.

Ten mice from the AS mouse model pretreatment group, 10 from the AS mouse model post-treatment group, and 10 normal mice were examined. The expression of the above genes in blood samples were detected by real-time PCR, and the results are shown in Fig. 2.

As shown in Fig. 2, the expression of TRAT1, CTLA4 and IL-15 in the model group before drug treatment were 1.6, 1.5, 1.5-fold of the control group, which was consistent with the screening results of differentially expressed genes $(\mathrm{P}<0.05)$. However, the expression of these genes were restored to normal levels after drug treatment, confirming that overexpression of these genes are associated with the occurrence of AS.

Protein levels of differentially expressed gene. To further study the relationship between these three genes and the disease, the protein expression levels of the genes in peripheral blood from 10 AS model mice before and after TNF treatment and 10 control group mice were detected by ELISA. The results are shown in Fig. 3. 
Table II. Primer sequence.

\begin{tabular}{lll}
\hline Gene & \multicolumn{1}{c}{ Forward primer F } & \multicolumn{1}{c}{ Reverse primer } \\
\hline TRAT1 & 5'-TGTAAGCTCGAGTTGTGGGCATCT-3' & 5'-TTCAGGAAGATTCGGGCACCTGAT-3' \\
CTLA4 & 5'-AGACCTGAACACCGCTCCCATAAAG-3' & 5'GCATTGCTTTGCAGAAGACAGGGA-3' \\
IL-15 & 5'-CCATGTGGCTCTTTGGAGCAATGT-3' & 5'-TCTTGTATGGGCTGGCTATCTGCT-3' \\
18sRNA & 5'-CCTGGATACCGCAGCTAGGA-3' & 5'-GCGGCGCAATACGAATGCCCC-3' \\
\hline
\end{tabular}

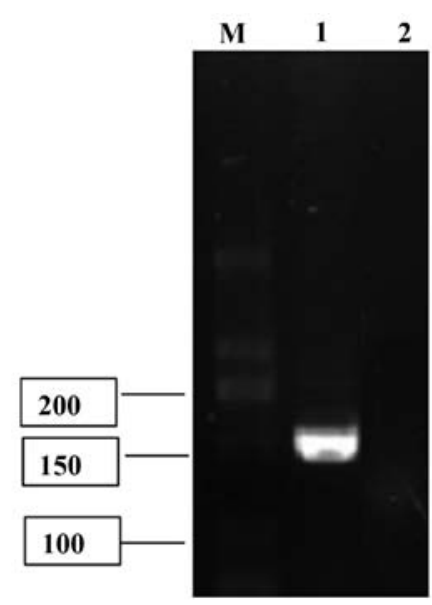

Figure 1. Expression of the HLA-B2704 gene in the peripheral blood by RT-PCR. M, positive group; 1, positive group; 2, control group.

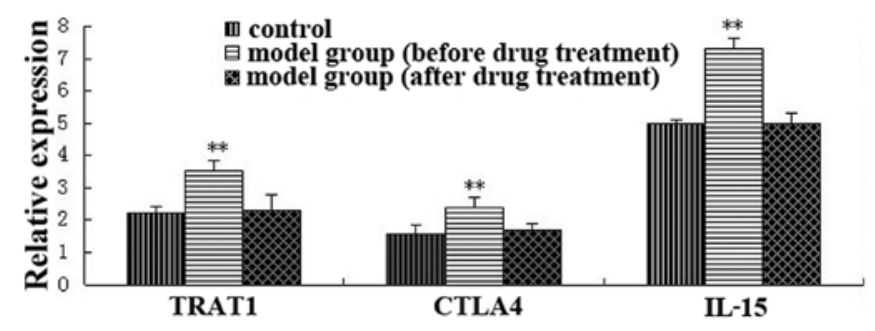

Figure 2. Expression of TRAT1, CTLA4, IL-15 in the control group and model group before and after treatment. ${ }^{* *}$ Represents significant difference.

As demonstrated in Fig. 3, consistent with the changes in gene expression, the protein (cytokine) level in the serum samples of the model group before drug administration was significantly higher than those of the control group. However, the protein levels were restored to normal levels after drug administration, suggesting that AS is not only associated with the mRNA expression of the three genes, but is also correlated with their protein expression levels.

Functional analysis of the CTLA4 gene. According to the screening results of differentially expressed genes, we then studied the role of CTLA4 in disease and its effect on Tlymphocyte activity under different concentrations. T lymphocytes were treated with different concentrations of CTLA4 protein, and the concentration of IL-5 in culture was examined. With the increase of CTLA4 concentration, the level of IL-5 was significantly reduced compared with the control group, and stabilized after reaching $90 \mathrm{ng} / \mathrm{ml}$ (Fig. 4).

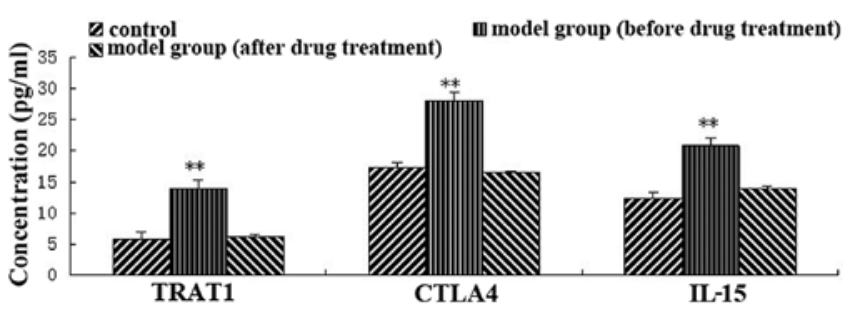

Figure 3. Protein levels of TRAT1, CTLA4, IL-15 in the control group and model group before and after treatment. ${ }^{* *}$ Represents significant difference.

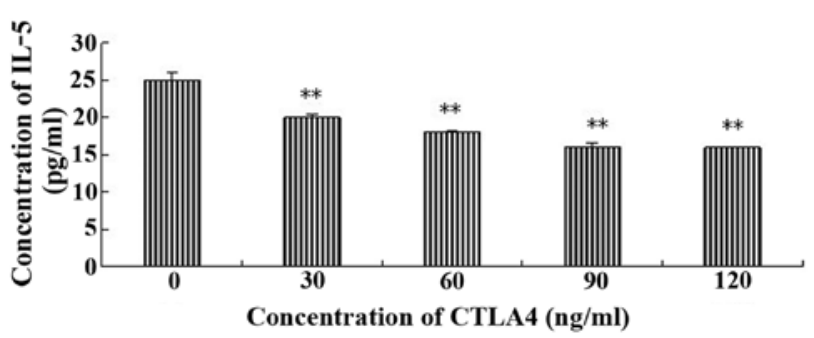

Figure 4. Effect of CTLA4 of different concentrations on IL-5 in T lymphocyte culture. * Represents significant difference.

\section{Discussion}

Ankylosing spondylitis (AS) is a progressive disease. Its first sign is pain in the unilateral sacroiliac joint and further leads to pain in the bilateral sacroiliac joint, lower legs, spines and if finally affects the ribs. In severe cases, thoracic expansion is impaired and cervical becomes mandatory, which limits the movement of the head and neck. Although the pathogenesis is not fully elucidated at present, many studies show that the occurrence of AS is closely related with hereditary genes, especially genes associated with the immune system. Studies have shown that, in patients suffering from AS, the expression of Th1 cytokines, such as TNF- $\alpha$ and IFN- $\gamma$ is significantly reduced, while adaptive immunity associated Th2 and Th17 expression is increased (6). In addition, many studies have demonstrated that the HLA-B27 gene is closely linked to AS, but the underlying mechanism remains unclear. Although HLA-B27 can hijack the $\mathrm{CD}^{+}$and $\mathrm{CD} 8^{+} \mathrm{T}$ cells that appear in joint fluid in aAS patients, it is possible that $\mathrm{CD} 4^{+}$and $\mathrm{CD} 8^{+}$ $\mathrm{T}$ cells act at the onset of the disease. Asides from HLA-B27, HLA-B60, HLA-DR1, CARD15, TFGF- $\beta$ as well as IL-2 are implicated in the occurrence of the disease $(3,4,7)$. We studied the disease at a genome-wide scale through differential gene expression profiling, obtaining related differentially expressed genes which underwent confirmation analyses. 
Cytotoxic T lymphocyte-associated antigen 4 (CTLA-4) is a transmembrane receptor on $\mathrm{T}$ cells, which shares $\mathrm{B} 7$ molecular ligand with CD28. Since the binding affinity between CTLA4 and B7 is 10-20 times higher than that of CD28 and B7, only a small quantity of CTLA4 can block the binding of CD28 and B7, inhibit T cell activation, and cause organic pathology, thus participating in negative regulation of the immune response (8). Takatsu (9) found that in T cellconditioned culture medium, IL-5 can replace the in vitro synergistic thymus-dependent antigen antibody responses of T cells, indicating that IL-5 is closely linked to T cell activity. Our study demonstrates that increasing TCLA4 concentration can significantly reduce $\mathrm{T}$ lymphocyte activity in the blood, suggesting that the CTLA4 gene may act through inhibition of T cell activity, thus leading to AS.

$\mathrm{T}$ cell receptor-related membrane ligand (TRAT1), a $29 \mathrm{kDa}$ dimer with disulfide bonds, consists of 159 amino acids, of which 3 tyrosine residues may participate in $\mathrm{SH} 2$-mediated intracellular molecular signaling. When $\mathrm{T}$ cells are activated, the TRAT1 protein can be phosphorylated rapidly and acts coordinately with the p58 regulatory subunit, presenting intracellular signals to the plasma membrane (10). At present, the function of TRAT1 has not been fully elucidated. Overexpression of TRAT1 has no effect on $\mathrm{T}$ cell receptor mediated activation of NAFT or IL-2 promoter transcriptional activity, which proves that TRAT1 may not regulate the occurrence of disease via the IL-2 pathway (11).

Interleukin 15 (IL-15) is mainly produced by mononuclear macrophage secretion, and exerts its biological function via the IL- 2 receptor $\beta$ chain and $\gamma$ chain (IL-2R $\beta, \gamma$ ). In addition, IL-15 possesses the specific IL-15a receptor chain, which is a high affinity IL-15 receptor chain. IL-15 can stimulate phytohemagglutinin-activated peripheral blood $\mathrm{T}$ cell proliferation and B cell secreting antibodies. Besides, IL-15 promotes NK cell proliferation and cytokine secretion, thus enhancing NK cell cytotoxic activity. The overexpression of IL-15 in tissues may lead to disordered immune system, triggering the occurrence of the disease.

Our study shows that AS pathogenesis is mainly related to immune-related genes, whose expression at both the mRNA and protein level is changed. Functional analysis identified that CTLA4 reduces IL-5 levels in T cell culture medium, suggesting that CTLA4 may act through inhibition of T cell activity, thus leading to AS pathogenesis. However, the mechanism of TRAT1 and IL-15 mediated AS pathogenesis remains to be further studied.

\section{References}

1. Assassi S, Reveille JD, Arnett FC, et al: Whole-blood gene expression profiling in ankylosing spondylitis shows upregulation of Toll-like receptor 4 and 5. J Rheumatol 38: 87-98, 2011.

2. Guo YY, Yang LL, Cui HD, Zhao S and Zhang N: Coexisting ankylosing spondylitis and rheumatoid arthritis: a case report with literature review. Chin Med J (Engl) 124: 3430-3432, 2011.

3. Howe HS, Cheung PL, Kong KO, et al: Transforming growth factor beta-1 and gene polymorphisms in oriental ankylosing spondylitis. Rheumatology 44: 51-54, 2005.

4. Kim TH, Uhm WS and Inman RD: Pathogenesis of ankylosing spondylitis and reactive arthritis. Curr Opin Rheumatol 17: 400-405, 2005

5. Spencer DJV: The involvement of HLA-B27 in ankylosing spondylitis. Medicine (SCJMM) 6: 84-89, 2005.

6. Szalay B, Meszaros G, Cseh A, et al: Adaptive immunity in ankylosing spondylitis: phenotype and functional alterations of T-cells before and during infliximab therapy. Clin Dev Immunol 2012: 808724, 2012

7. George RA, Liu JY, Feng LL, Bryson-Richardson RJ, Fatkin D and Wouters MA: Analysis of protein sequence and interaction data for candidate disease gene prediction. Nucleic Acids Res 34: e130, 2006

8. Oosterwegel MA, Greenwald RJ, Mandelbrot DA, Lorsbach RB and Sharpe AH: CTLA-4 and T cell activation. Curr Opin Immunol 11: 294-300, 1999.

9. Takatsu K: Interleukin 5 and $\mathrm{B}$ cell differentiation. Cytokine Growth Factor Rev 9: 25-35, 1998.

10. Bruyns E, Marie-Cardine A, Kirchgessner H, et al: T cell receptor (TCR) interacting molecule (TRIM), a novel disulfide-linked dimer associated with the TCR-CD3-zeta complex, recruits intracellular signaling proteins to the plasma membrane. J Exp Med 188: 561-575, 1998.

11. Zhang W and Samelson LE: The role of membrane-associated adaptors in T cell receptor signalling. Semin Immunol 12: 35-41, 2000 . 